\title{
Antikaon Production and Medium Effects in Heavy Ion Collisions at AGS
}

\author{
Guang Song ${ }^{1}$, Bao-An $\mathrm{Li}^{2}{ }^{*}$, and C. M. $\mathrm{Ko}^{1 \dagger}$ \\ ${ }^{1}$ Cyclotron Institute and Physics Department, \\ Texas A\&M University, College Station, TX 77843, USA \\ 2 Department of Chemistry and Physics, \\ Arkansas State University, State University, AR 72467, USA
}

\begin{abstract}
Antikaon production from heavy ion collisions at energies available from the Alternating Gradient Synchrotron (AGS) at the Brookhaven National Laboratory is studied in a relativistic transport (ART) model. We include contributions from the baryon-baryon, meson-baryon, and meson-meson interactions. The final-state interaction of antikaons via both absorption and elastic scattering by nucleons and pions are also considered. To compare with presently available or future experimental data, we have calculated the antikaon rapidity and transverse momentum distributions as well as its collective flow. Medium effects on these observables due to mean field potentials have also been investigated. It is found that the ratio of antikaon transverse momentum spectrum to that of kaon and their transverse flow are most sensitive to the in-medium properties of kaons and antikaons.
\end{abstract}

PACS: 25.75.-q

Keywords: Antikaon; Relativistic heavy ion collisions; Medium effects

*E-mail: Bali@navajo.astate.edu

†E-mail: ko@comp.tamu.edu 


\section{INTRODUCTION}

For heavy ion collisions at the AGS energies, transport model studies [1] have indicated that both density and temperature of the participant region are high. Heavy ion experiments at AGS thus offer the possibility to study not only the hadron to quark-gluon plasma transition but also the properties of hadrons, such as their masses and lifetimes, in dense medium 24 [4. Such medium effects have recently attracted much attention as they may be related to the precursor effects due to chiral symmetry restoration [5, 6]. In particular, knowledge on the properties of kaons in the nuclear medium is important for understanding both chiral symmetry restoration and neutron star properties. Since the suggestion by Kaplan and Nelson on the possibility of kaon condensation in dense matter [7], there have been many theoretical studies on the in-medium properties of kaons, using various models, such as the chiral Lagrangian [8], the boson exchange model [9, 10], the Nambu-Jona-Lasino model [11], and the coupled-channel approach [12,[13]. These studies have all shown that $K^{+}$ feels a weak repulsive potential while $K^{-}$sees a strong attractive potential in the nuclear medium. As a result, a condensation of antikaons in neutron stars becomes plausible [7, 14], which would then lead to the possible existence of many mini black holes in the galaxies [15,16].

A number of experiments have recently been carried out at the AGS [17], and preliminary data from these experiments seem to indicate that medium effects associated with kaons are already present in some of the observed phenomena [18]. While the analysis of experimental data is being finalized, a critical theoretical examination of both the production mechanism for $K^{+}$and $K^{-}$and the medium effects on experimental observables will be very useful. For $K^{+}$, we have already used the ART model [1] to study its production in heavy ion collisions at AGS and the dependence of its momentum spectra on its in-medium properties [19]. In particular, we have found that the $K^{+}$transverse collective flow is sensitive to the kaon dispersion relation in dense nuclear matter. In the present work, we shall report a similar study for $K^{-}$.

In Section II, we will briefly describe the ART model used in our previous study of particle production and signatures of chiral symmetry restoration and/or QGP formation in heavy ion collisions at AGS energies [1, 19,20]. We will then discuss the details of implementing various reaction channels for $K^{-}$production. These include $K^{-}$production from baryon-baryon, meson-baryon, and meson-meson interactions. Also, $K^{-}$absorption and its final-state elastic scattering will be considered. In Section III, results from this study are presented. In particular, we shall discuss the relative contributions of different reaction channels to the $K^{-}$yield, its rapidity and transverse mass distribution and collective flow. Medium effects due to both the nuclear and Coulomb potentials on these observables will also be investigated. The beam energy dependence of the medium effects on both $K^{+}$and $K^{-}$will be studied. Finally, a summary is given in Section IV.

\section{ANTIKAON PRODUCTION, ABSORPTION AND RESCATTERING IN THE ART MODEL}

The ART model is a pure hadronic transport model developed for modeling relativis-

tic heavy ion collisions up to the AGS energies [1]. For completeness, we summarize here 
the main features of this model and refer the reader to Ref. [1] for its details. The ART 1.0 includes the following baryons: $N, \Delta(1232), N^{*}(1440), N^{*}(1535), \Lambda, \Sigma$; and mesons: $\pi, K, \eta, \rho, \omega$; as well as their explicit charge states. Both elastic and inelastic collisions among most of these particles are modeled as best as we can by using as inputs the experimental data from hadron-hadron collisions. Most inelastic hadron-hadron collisions are modeled through the formation of baryon and meson resonances. Although we have only explicitly included three baryon resonances, effects of heavier baryon resonances with masses up to $2 \mathrm{GeV}$ are partially taken into account through the formation of these resonances in the intermediate states of meson-baryon reactions. We have also included in the model optional, self-consistent mean field potentials for both baryons and kaons.

The treatment of antikaon in the ART model 1.0 is, however, incomplete as only antikaon production from meson-meson interactions has been included. Although this has negligible effects on the reaction dynamics and experimental observables associated with nucleons, pions and kaons, we have been unable to study in detail the production mechanism for antikaon and its dependence on the medium effects. In this section, we shall first discuss the improvement we have made in the ART model for treating antikaon production, absorption, rescattering, and propagation.

\section{A. Antikaon production from baryon-baryon interactions}

There are few experimental data on $K^{-}$production from nucleon-nucleon interactions in the energy range we are considering. In particular, its total production cross section from $p p$ collisions is practically unknown. There are a number of parameterizations of the antikaon production cross section from the nucleon-nucleon interaction [21 24]. For example, the inclusive $K^{-}$production cross section from the proton-proton interaction, i.e., $p p \rightarrow K^{-} X$, has been parameterized in Ref. [21] using phase space consideration. We choose this one in the present work as it fits better the available data at high energies. Specifically, the $K^{-}$ production cross section from $p p$ collisions is given by

$$
\sigma_{p p \rightarrow K^{-X}}(s)=\left(1-\frac{s_{0}}{s}\right)^{3}\left[2.8 F_{1}\left(\frac{s}{s_{0}}\right)+7.7 F_{2}\left(\frac{s}{s_{0}}\right)\right]+3.9 F_{3}\left(\frac{s}{s_{0}}\right)[\mathrm{mb}],
$$

with

$$
\begin{aligned}
& F_{1}(x)=(1+1 / \sqrt{x}) \ln (x)-4(1-1 / \sqrt{x}), \\
& F_{2}(x)=1-(1 / \sqrt{x})(1+\ln (x) / 2), \\
& F_{3}(x)=\left(\frac{x-1}{x^{2}}\right)^{3.5} .
\end{aligned}
$$

In the above, $s_{0}{ }^{1 / 2}=2\left(m_{p}+m_{K}\right)=2.8639 \mathrm{GeV}$ is the threshold energy.

At AGS energies the final state is expected to be dominated by two nucleons and a kaon-antikaon pair. We thus assume that the cross section for $p p \rightarrow p p K^{+} K^{-}$is the same as that for $p p \rightarrow K^{-} X$. Since there are no data for $K^{-}$production from $n p, n n$ and other baryon-baryon interactions involving one or two resonances, to determine their cross sections thus requires models for these interactions. In the present study, we make the minimum 
assumption that they all have the same $K^{-}$production cross section as in $p p$ collisions at the same center of mass energy.

To determine the momentum distribution of the produced $K^{-}$, we make use of the empirical observation that the momentum distributions of final particles in high energy $p p$ collisions all have the following form

$$
\frac{d^{2} \sigma}{d p_{T}^{2} d p_{L}} \propto e^{-A x^{* 2}} e^{-B p_{T}^{2}},
$$

where $x^{*}=p_{\mathrm{L}} / p_{\mathrm{L}_{\max }}$ with $p_{\mathrm{L}_{\max }}$ being the maximum longitudinal momentum of the particle. In the ART model, this is carried out by first obtaining the $K^{-}$and $K^{+}$transverse and longitudinal momenta from the above distribution, assuming that the angle between $p_{x}$ and $p_{y}$ is uniformly distributed. Then, the longitudinal momenta of the two baryons are obtained using also a similar distribution. From energy and momentum conservation, the transverse momenta of both baryons can be determined. We find that the limited experimental data 25] are reasonably fitted by using the following values: $A=12.5$ and $B=4.15(\mathrm{GeV} / \mathrm{c})^{2}$ for $K^{-} ; A=5.3$ and $B=3.68(\mathrm{GeV} / \mathrm{c})^{2}$ for $K^{+}$; and $A=2.76(\mathrm{GeV} / \mathrm{c})^{2}$ for $N$.

\section{B. Antikaon production from meson-baryon interactions}

The cross section for $K^{-}$production from pion-nucleon interactions has been studied in Ref. 22] using a boson-exchange model. Reactions with one or more pions in the final state are neglected as their cross sections are small in the energy range we consider. Following Ref. [22], we have

$$
\begin{aligned}
2 \sigma\left(\pi^{-} p \rightarrow p K^{0} K^{-}\right) & =\sigma\left(\pi^{-} p \rightarrow n K^{+} K^{-}\right)=\sigma\left(\pi^{-} n \rightarrow n K^{0} K^{-}\right) \\
= & 4 \sigma\left(\pi^{0} p \rightarrow p K^{+} K^{-}\right)=4 \sigma\left(\pi^{0} n \rightarrow n K^{+} K^{-}\right)=\sigma\left(\pi^{0} n \rightarrow p K^{0} K^{-}\right) \\
=\sigma\left(\pi^{+} n \rightarrow p K^{+} K^{-}\right) & =\sigma_{0},
\end{aligned}
$$

where $\sigma_{0}$ is given by 22

$$
\sigma_{0}=1.21\left(1-s_{0} / s\right)^{1.86}\left(s_{0} / s\right)^{2}[\mathrm{mb}] .
$$

For $\pi$-baryon resonance and $\rho(\omega)$-baryon collisions there are no experimental data. We again make the minimum assumption that their cross sections are the same as that in pionnucleon interaction at the same center of mass energy.

The momentum distribution of the produced $K^{-}$from meson-baryon interactions is determined by the three-body phase space.

\section{Antikaon production from meson-meson interactions}

As in the original ART model [1], antikaon productions from all meson-meson collisions are modeled through the process $M M \rightarrow K \bar{K}$. The cross section used for $\pi \pi \rightarrow K \bar{K}$ is calculated from the $K^{*}$ exchange model of Ref. [2]. All other meson-meson reactions, such as $\rho \rho \rightarrow K \bar{K}$ or $\pi \rho \rightarrow K \bar{K}$, are taken to have a constant value of $0.3 \mathrm{mb}$. Contrary to antikaon production from baryon-baryon and meson-baryon interactions, the momentum of

produced antikaon from meson-meson interaction is trivially fixed by kinematics as the final state consists of only two particles. 


\section{Antikaon absorption and its production from meson-hyperon interactions}

Antikaons produced in hot dense matter can be absorbed by nucleons via strangeexchange reactions. For final states consisting of a $\Sigma$ particle, we have the following reactions:

$$
\begin{aligned}
& K^{-} p \rightarrow \Sigma^{0} \pi^{0}, \Sigma^{-} \pi^{+}, \Sigma^{+} \pi^{-}, \\
& K^{-} n \rightarrow \Sigma^{0} \pi^{-}, \Sigma^{-} \pi^{0} .
\end{aligned}
$$

Their cross sections are taken from Ref. [26], i.e.,

$$
\begin{gathered}
\sigma\left(K^{-} p \rightarrow \Sigma^{0} \pi^{0}\right)=0.6 p^{-1.8}[\mathrm{mb}] ; 0.2 \leq p \leq 1.5 \mathrm{GeV} / \mathrm{c} \\
\sigma\left(K^{-} n \rightarrow \Sigma^{0} \pi^{-}\right)=\left\{\begin{array}{l}
1.2 p^{-1.3}[\mathrm{mb}], \text { if } 0.2 \leq p \leq 1 \mathrm{GeV} / \mathrm{c} \\
1.2 p^{-2.3}[\mathrm{mb}], \text { if } 1 \leq p \leq 6 \mathrm{Gev} / \mathrm{c} .
\end{array}\right.
\end{gathered}
$$

where $p$ is the $K^{-}$momentum in the laboratory frame.

For $K^{-}$absorption by resonances, we take the cross sections for $N^{*+}$ and $\Delta^{+}$to be the same as for $p$, while those for $N^{* 0}$ and $\Delta^{0}$ the same as for $n$. We also include the reactions $K^{-} \Delta^{++} \rightarrow \Sigma^{+} \pi^{0}, \Sigma^{0} \pi^{+}$by assuming that their cross sections are the same as that for $K^{-} p$. On the other hand, the cross section for $K^{-} \Delta^{-} \rightarrow \Sigma^{-} \pi^{-}$is taken to be the same as that for $K^{-} n$.

For final states with a $\Lambda$, the following reactions are possible,

$$
\begin{aligned}
& K^{-} p \rightarrow \Lambda+\pi^{0}, \\
& K^{-} n \rightarrow \Lambda+\pi^{-} .
\end{aligned}
$$

The $K^{-} \mathrm{p}$ cross section is parameterized as in Ref. [26], i.e.,

$$
\sigma_{K^{-} p \rightarrow \Lambda \pi^{0}}= \begin{cases}50 p^{2}-67 p+24[\mathrm{mb}], & \text { if } 0.2 \leq p \leq 0.9 \mathrm{GeV} \\ 3 p^{-2.6}[\mathrm{mb}], & \text { if } \quad 0.9 \leq p \leq 10 \mathrm{GeV}\end{cases}
$$

For other cross sections involving $n$ and baryon resonances, they are assumed to be the same as $K^{-} p \rightarrow \Lambda \pi^{0}$.

In the inverse reactions, i.e. $\pi+\Lambda(\Sigma)$, antikaon can be reproduced, and their cross sections are deduced from the antikaon absorption cross section using the detailed balance relations. A hyperon can also interact with other mesons, such as rho and omega to produce a $K^{-}$. The corresponding cross sections are assumed to be the same as those for a pion at the same center of mass energy.

\section{E. Antikaon final-state interactions}

Because of final-state interactions, not all antikaons produced in hadron-hadron interactions can escape freely from the reaction zone in heavy ion collisions. The absorption reactions have already been described in the previous section. Here, we are mainly concerned with the $K^{-}$elastic scatterings with baryons. These cross sections are taken from Ref. [26], i.e., 


$$
\begin{aligned}
& \sigma_{K^{-} p \rightarrow K^{-} p}=13 p^{-0.9}[\mathrm{mb}], 0.25 \leq p \leq 4.0 \mathrm{GeV} / \mathrm{c} \text {. } \\
& \sigma_{K^{-} n \rightarrow K^{-} n}= \begin{cases}20.0 p^{-2.74}[\mathrm{mb}], & \text { if } 0.5 \leq p \leq 1.0 \mathrm{GeV} / \mathrm{c} \\
20.0 p^{-1.8}[\mathrm{mb}], & \text { if } 1.0<p \leq 4.0 \mathrm{GeV} / \mathrm{c}\end{cases}
\end{aligned}
$$

\section{F. Mean field potentials for kaons and antikaons}

In transport models, the imaginary part of the self energy of a hadron is approximately treated by its scatterings with other hadrons, while the real part of the self energy is given by the mean field potential. Various approaches have been used to evaluate the kaon mean field potential in the nuclear medium [8 13], we use here the one determined from the kaonnucleon scattering length $a_{K N}$ in the impulse approximation [16], i.e.,

$$
\omega\left(p, \rho_{b}\right)=\left[m_{K}^{2}+p^{2}-4 \pi\left(1+\frac{m_{K}}{m_{N}}\right) a_{K N} \rho_{b}\right]^{1 / 2},
$$

where $m_{K}$ and $m_{N}$ are the kaon and nucleon masses, respectively; $p$ is the kaon momentum; $\rho_{b}$ is the baryon density; and $a_{K N} \approx-0.255 \mathrm{fm}$ is the isospin-averaged kaon-nucleon scattering length. The kaon potential in the nuclear medium is given by

$$
U\left(p, \rho_{b}\right)=\omega\left(p, \rho_{b}\right)-\left(m_{K}^{2}+p^{2}\right)^{1 / 2}
$$

At normal nuclear density, a kaon at rest has a repulsive potential of about $30 \mathrm{MeV}$.

For the $K^{-}$potential, we use the following expression

$$
U(\rho)=-0.12\left(\rho_{b} / \rho_{0}\right)[\mathrm{GeV}]
$$

The magnitude of this potential is similar to that either extracted from the experimental data on kaonic atom or predicted by theoretical models 8 13.

One of the main purposes of this work is to explore the effects of kaon potentials on experimental observables. We shall thus compare in the next section results from calculations with and without mean field potentials for kaons and antikaons. Although the model at the present stage probably cannot distinguish the different forms of kaon and antikaon potentials from various theories, it does predict that some observables are appreciably affected by the presence of mean field potentials as shown below.

\section{RESULTS AND DISCUSSIONS}

We now turn to the results of our study. After including all $K^{-}$production channels outlined above, our previous predictions, that are based on the ART 1.0, for the nucleon, pion and kaon observables do not show significant changes as antikaons are small perturbations to the collision dynamics and other hadrons. We thus only present in the following results for antikaons. 


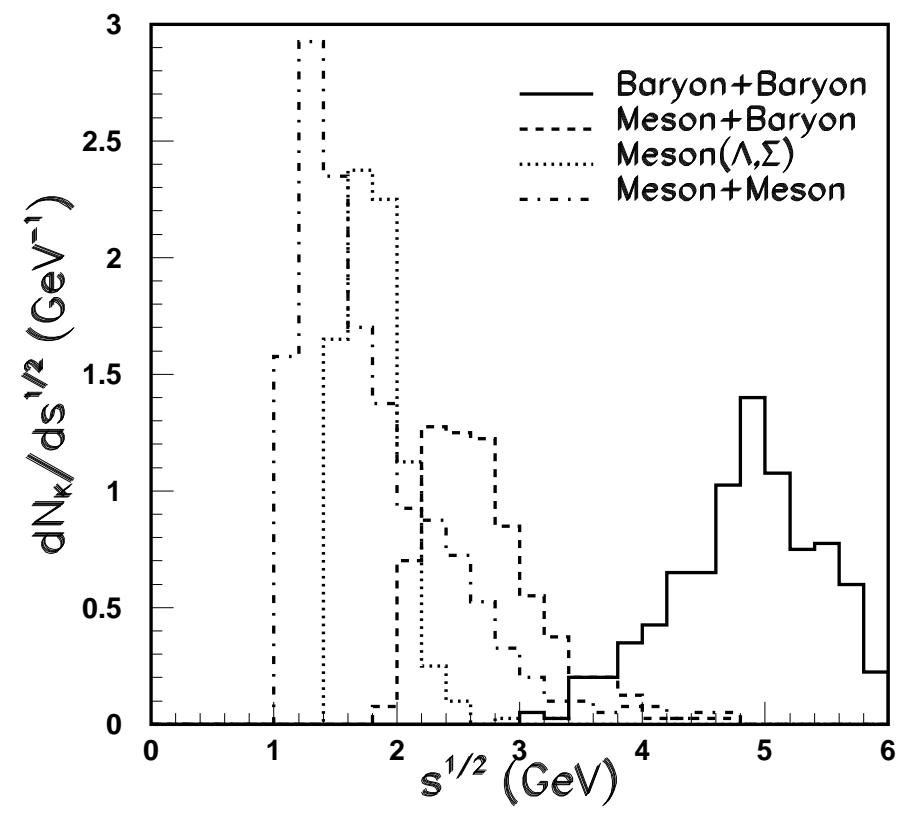

FIG. 1. Center of mass energy distribution of hadron pairs with energies above the $K^{-}$production threshold in the reaction of $\mathrm{Au}+\mathrm{Au}$ at $p_{\text {beam }} / \mathrm{A}=11.6 \mathrm{GeV} / \mathrm{c}$ and impact parameter $\mathrm{b}$ $=4 \mathrm{fm}$, and with the $K^{-}$mean field potential.

\section{A. Production mechanism for antikaons}

To identify the sources for antikaon production, we show in Fig. 1 the center of mass energy distribution of hadron pairs with energies above the $K^{-}$production threshold in the reaction of $\mathrm{Au}+\mathrm{Au}$ at $p_{\text {beam }} / \mathrm{A}=11.6 \mathrm{GeV} / \mathrm{c}$ and impact parameter of $4 \mathrm{fm}$. It is seen that contributions from meson-meson and meson-baryon collisions are more important than that from baryon-baryon collisions. More quantitatively, about 40\%, $40 \%$ and $20 \%$ of produced $K^{-}$are from meson-meson, meson-baryon, and baryon-baryon collisions, respectively. The relative importance of different $K^{-}$sources seen here thus does not agree completely with results from either RQMD [27], where meson-baryon interactions seem to contribute most, or the ARC 28] model, which shows a main contribution from baryon-baryon interactions. We attribute the different conclusions to the fact that different assumptions about the cross sections for the elementary $K^{-}$production reactions are introduced in these models. Similar differences have also been seen previously in transport model studies of $K^{+}$production [1].

To learn about the dynamics of antikaon production, the primordial $K^{-}$multiplicity is shown in Fig. 2 as a function of time for various $K^{-}$production channels by turning off the $K^{-}$absorption reactions in the calculation. It is seen that $K^{-}$production starts at about $\mathrm{t}=0.5 \mathrm{fm} / \mathrm{c}$ after the contact of the colliding nuclei and is initially dominated by the contribution from baryon-baryon collisions. Soon after that, meson-baryon collisions begin to contribute significantly. The meson-meson collisions do not contribute until around 2 $\mathrm{fm} / \mathrm{c}$ and become the dominant ones after $4 \mathrm{fm} / \mathrm{c}$. Both $K^{-}$production and absorption from meson-meson collisions and their inverse reactions are seen to last longer than all other reactions. We note that $K^{-}$production from baryon-baryon collisions practically ceases 
after about $4 \mathrm{fm} / \mathrm{c}$.

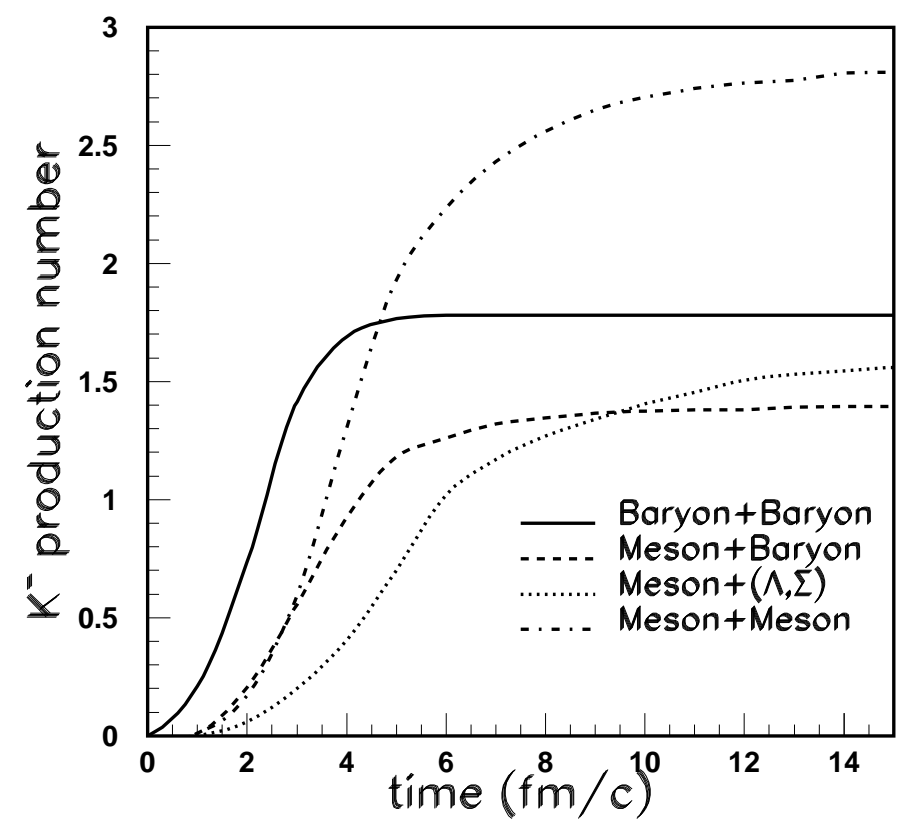

FIG. 2. Primordial $K^{-}$number from different reaction channels as functions of time in the same reaction as in Fig. 1.

Including $K^{-}$absorption we have repeated the calculations for the same reaction as in Fig. 2, and the results are shown in Fig. 3. Comparing Fig. 2 and Fig. 3 one sees that close to half of the $K^{-}$'s produced from baryon-baryon collisions are absorbed during their propagation through the system. For both meson-baryon and meson-hyperon collisions, about $40 \%$ of the primordial $K^{-}$are absorbed, while it is only about one fourth for those created through meson-meson collisions. This is mainly because $K^{-}$'s from baryon-baryon collisions are produced earlier in the collisions, so they spend a longer time in the dense matter and thus have a higher chance to get absorbed. Another reason is that $K^{-}$'s from baryon-baryon collisions generally have higher kinetic energies and thus have velocities comparable to those of baryons, so it is easier for them to be absorbed.

\section{B. Mean field effects on antikaon spectra and yields}

To study the effects of mean field potentials on kaons and antikaons, we have calculated their transverse mass spectra and rapidity distributions with and without mean field potentials. We shall also compare them with recent data from the E802/E866 collaboration [29].

Fig. 4 shows the $K^{+}$and $K^{-}$transverse mass distributions from the reaction of $\mathrm{Au}+$ $\mathrm{Au}$ at $p_{\text {beam }} / \mathrm{A}=11.6 \mathrm{GeV} / \mathrm{c}$ and impact parameters $b \leq 4 \mathrm{fm}$. It is seen that the attractive mean field potential pulls $K^{-}$to lower values of transverse momentum, causing its slope to increase, while for $K^{+}$, the effect seems to be opposite. Since the mean field potential is stronger for $K^{-}$than for $K^{+}$, the effect is also larger for $K^{-}$than for $K^{+}$. While the $K^{+}$ 


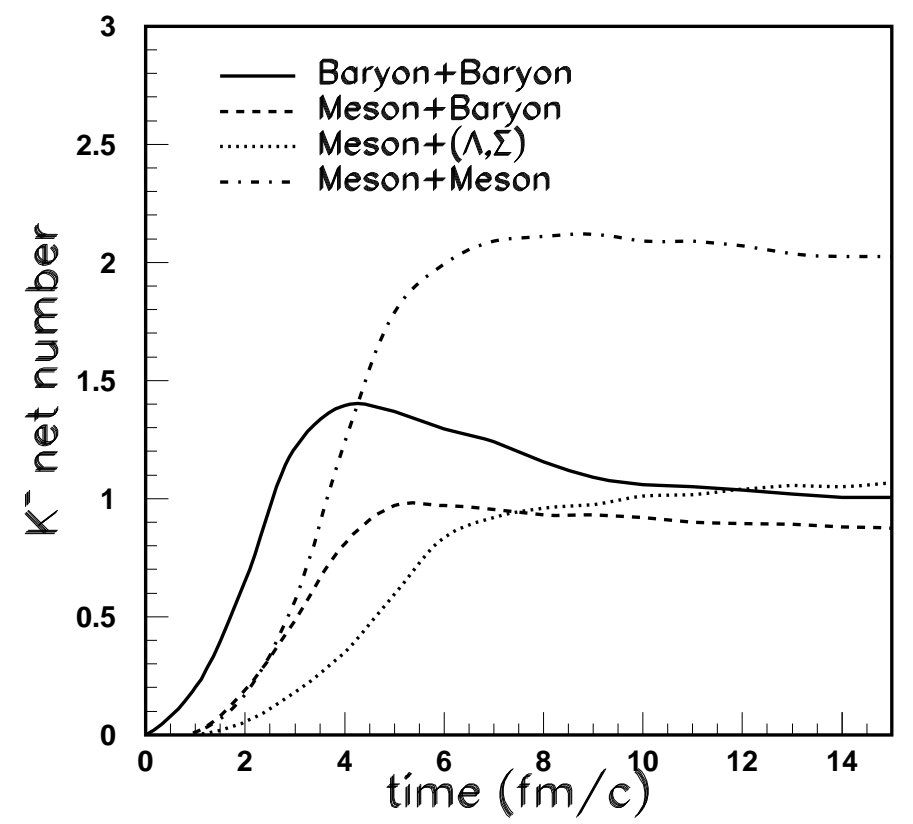

FIG. 3. Net $K^{-}$number after absorption for the same reaction as in Fig. 1.

data can be reproduced reasonably well by both calculations with and without the mean field potential, the calculations with the mean field potential for $K^{-}$seem to better reproduce the data.

It has been proposed that the $K^{+} / K^{-}$ratio as a function of their transverse mass is a sensitive probe of mean field effects [19]. This is because the average thermal velocities of $K^{+}$and $K^{-}$, which are much larger than the change of velocity caused by medium effects, are almost canceled out. Indeed, as shown in Fig. 5 for the same reaction as in Fig. 4 this ratio decreases with the transverse mass $m_{\mathrm{t}}$ in the case without mean field potentials but increases with $m_{\mathrm{t}}$ once mean field potentials are included.

The $K^{+}$and $K^{-}$rapidity distributions for the same $\mathrm{Au}+\mathrm{Au}$ reaction as in Fig. 4 are shown in Fig. 6. The rapidity distribution for $K^{-}$is seen to be narrower than that for $K^{+}$. This may also be a signature of the mean field effect as the strong attractive $K^{-}$mean field potential makes $K^{-}$less energetic, leading thus to a narrower rapidity distribution around the mid-rapidity than in the case without a potential. Although these low energy $K^{-}$'s at the central rapidity are more easily absorbed by nucleons, the inverse reactions of $K^{-}$production from pion-hyperon interactions is also more important as most pions and hyperons are concentrated at the central rapidity. One thus expects an increase of the $K^{-}$ yield after including the mean field potential. The theoretical results support such a picture. Indeed, without mean field potentials the rapidity distribution for $K^{+}$is narrower than that for $K^{-}$but becomes much wider after the mean field potentials are included. Also, the increase in the $K^{-}$yield at central rapidity is more than its decrease at the projectile and target rapidities, leading to an increased total $K^{-}$yield when the mean field potential is introduced. This effect can be more clearly seen in Fig. 7 where the ratio $K^{+} / K^{-}$is shown as a function of rapidity $y$. It shows that the ratio decreases from about 4.2 around the 


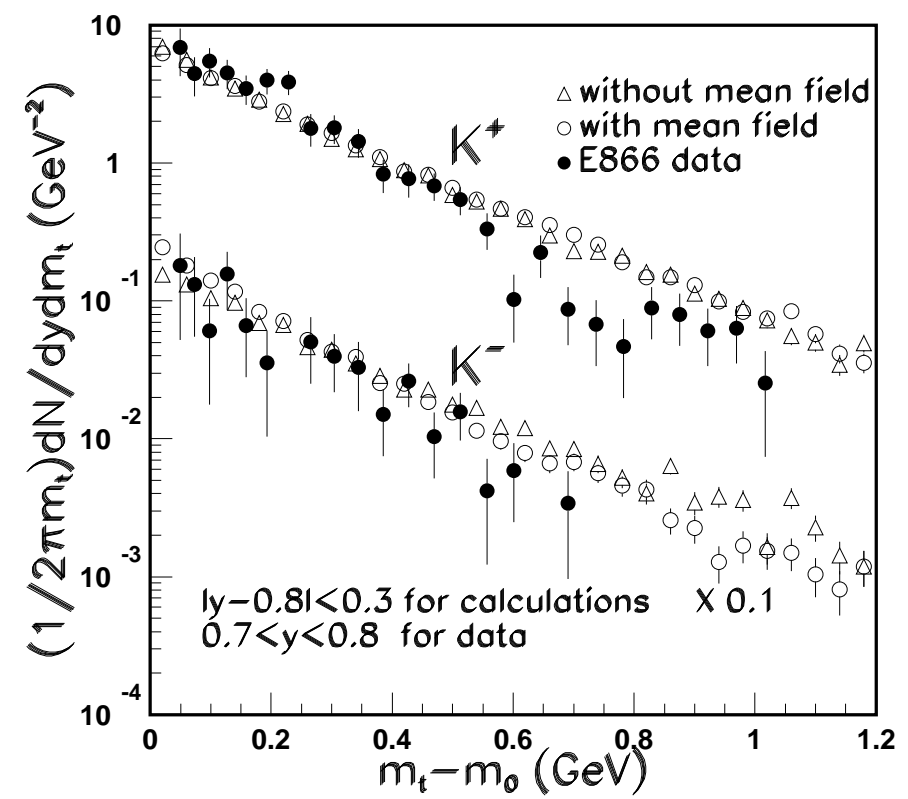

FIG. 4. Transverse mass spectra of $K^{+}$and $K^{-}$in the reaction of $\mathrm{Au}+\mathrm{Au}$ at $p_{\text {beam }} / \mathrm{A}=11.6$ $\mathrm{GeV} / \mathrm{c}$ and impact parameters $b \leq 4 \mathrm{fm}$.

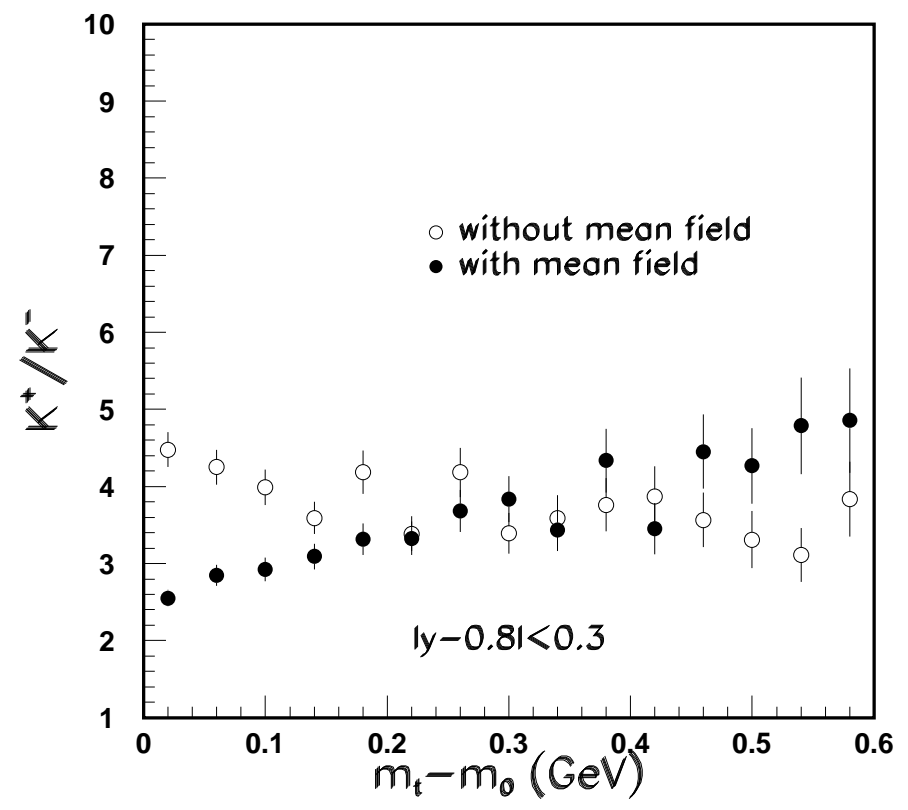

FIG. 5. Transverse mass spectra of $K^{+} / K^{-}$in the same reaction as in Fig. 4. 


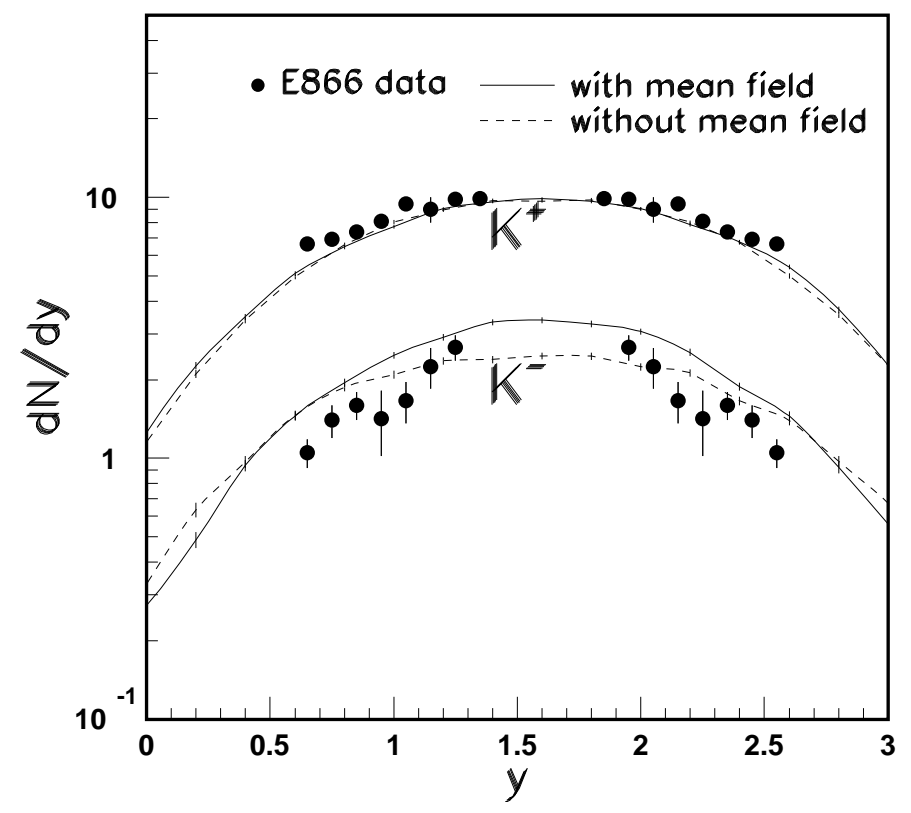

FIG. 6. Rapidity distributions of $K^{+}$and $K^{-}$in the same reaction as in Fig. 4.

mid-rapidity to about 3.5 around the target and projectile rapidities if no kaon mean field is included. Medium effects change this ratio dramatically; it increases from about 3.0 at the mid-rapidity to about 4.0 at the target and projectile rapidities.

We note that both $K^{+}$and $K^{-}$production from $\mathrm{Au}+\mathrm{Au}$ collisions at $p_{\text {beam }} / \mathrm{A}=11.6$ $\mathrm{GeV} / \mathrm{c}$ have also been studied in the HSD model [30], which includes both initial string dynamics and subsequent hadron cascade. Contrary to our results as well as those from the ARC and RQMD models, the HSD model underpredictes the yield of both $K^{+}$and $K^{-}$. This may be due to the introduction in the model a finite formation time, which is not included in either our model or the ARC model. Although finite formation time is included in the RQMD model, the resulting large yield of $K^{+}$and $K^{-}$is probably due to the inclusion of high mass resonances, which are neglected in the HSD model. More work is thus required to clarify the effects due to different physical assumptions in these transport models.

\section{Transverse flow analysis for antikaons}

It was first demonstrated in Ref. 31 that kaon transverse flow is a powerful probe of kaon in-medium potentials in heavy ion collisions at SIS/GSI energies, which are an order of magnitude lower than that at AGS. Subsequently, using ART 1.0 the kaon transverse flow has also been found to be the most sensitive observable for studying the kaon dispersion relation in the dense medium formed in relativistic heavy ion collisions at AGS energies [19. It is thus interesting to compare the transverse flow of kaons and antikaons as their mean field potentials have opposite signs.

First, we perform the standard transverse flow analysis for $K^{-}$for the same $\mathrm{Au}+\mathrm{Au}$ reaction as in Fig. 4, and the results are shown in Fig. 8. It is seen that without $K^{-}$ 


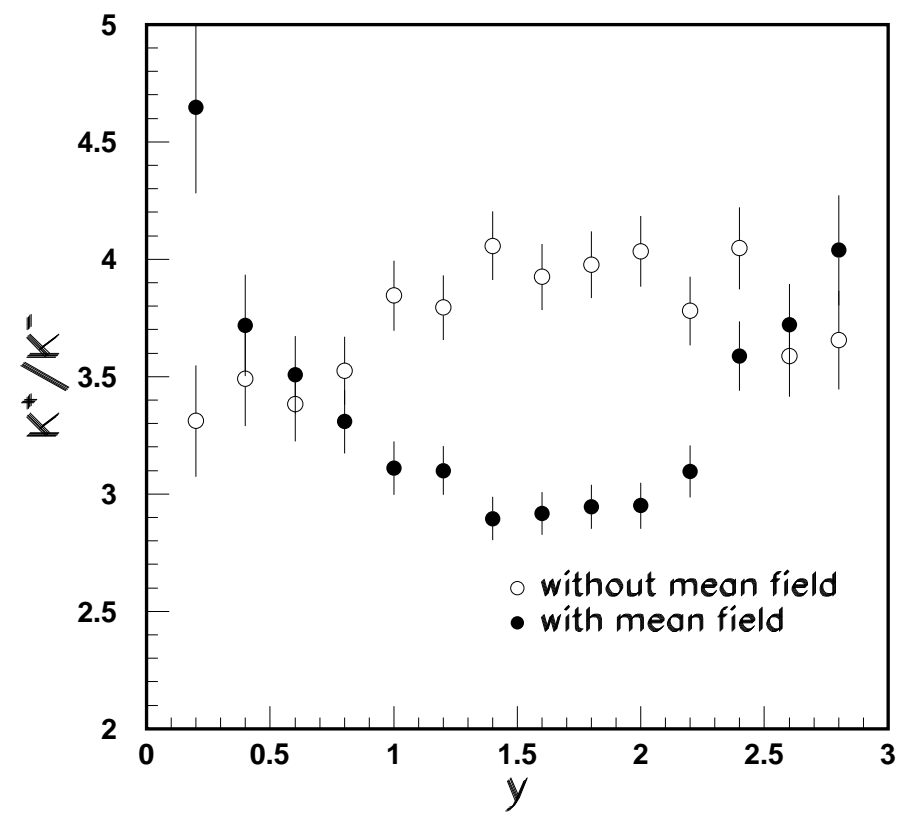

FIG. 7. Rapidity distributions of the $K^{+} / K^{-}$ratio in the same reaction as in Fig. 4.

potential (shown by open circles) antikaons flow in the opposite direction as that of nucleons since antikaons flowing with nucleons are absorbed due to strong strange-exchange reactions. Such a shadowing effect due to the spectator matters has also been seen in the transverse flow of pions 32 35. However, when the attractive mean field potential is included (shown by solid circles), the flow of antikaons change its direction toward that of nucleons.

For comparison we show in Fig. 9 the transverse flow of $K^{+}$calculated with and without mean field potentials for the same $\mathrm{Au}+\mathrm{Au}$ reaction as in Fig. 8. For both $K^{+}$and $K^{-}$, their transverse flow are reversed once mean field potentials are introduced in the transport model. The transverse flow of antikaons is, however, found to be more sensitive to the mean field potential due to the stronger antikaon potential compared to that for kaon. This makes antikaon flow analysis an even more valuable tool for studying the in-medium properties of antikaons. It is interesting to mention that the preliminary data from the E866 collaboration on the $K^{+}$and $K^{-}$flow is consistent with those predicted by an attractive mean field potential for $K^{-}$and a repulsive one for $K^{+}$36.

\section{Coulomb effects on the transverse momentum spectra of kaons and antikaons}

Besides the mean field potential due to strong interaction, both $K^{+}$and $K^{-}$are also affected by their Coulomb potentials. While the mean field potential is attractive and repulsive for $K^{-}$and $K^{+}$, respectively, the Coulomb potentials have similar effects. To see the relative importance of mean field and Coulomb potentials on the spectra of $K^{+}$and $K^{-}$, the following four different calculations have been carried out: with Coulomb potential only, with mean field potential only, with both potentials, and without any potential. Results of these studies are shown in Fig. 10 for the same reaction as in Fig. 4. It is seen that for $\mathrm{K}^{-}$ 


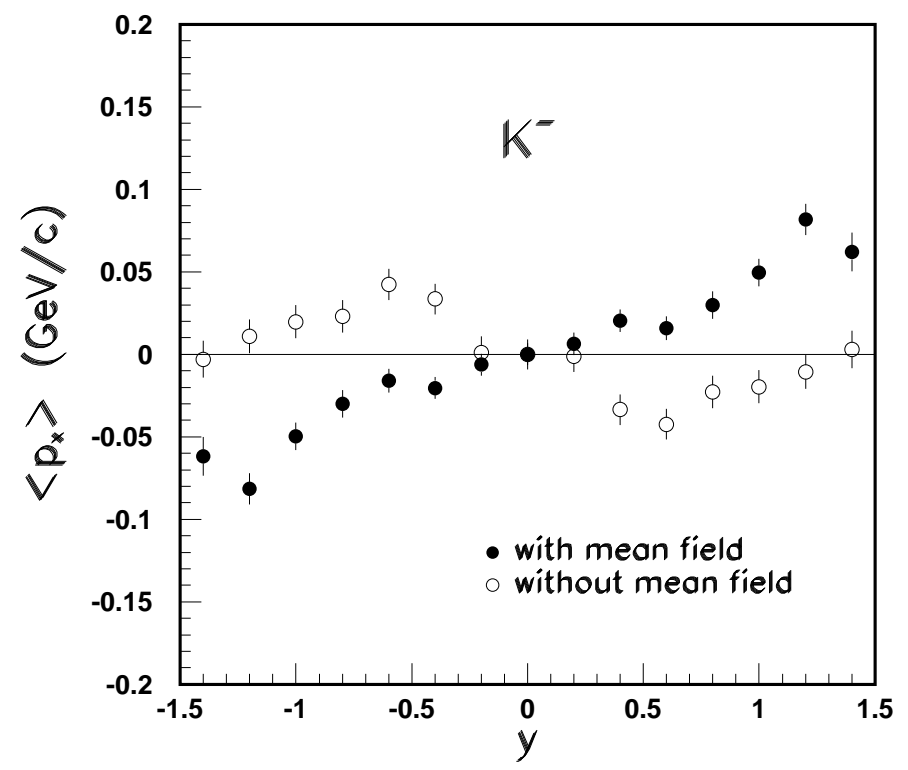

FIG. 8. Transverse flow of $K^{-}$in the same reaction as in Fig. 4.

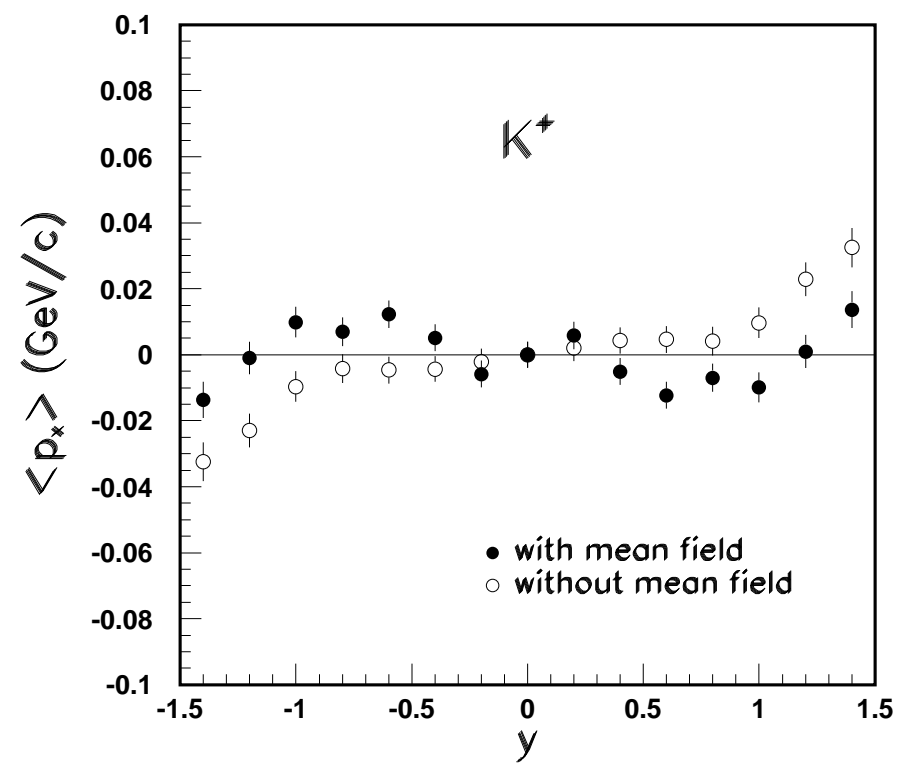

FIG. 9. Transverse flow of $K^{+}$in the same reaction as in Fig. 4. 
the effect due to the mean field potential is much stronger than that due to the Coulomb potential. This is different from the $K^{+}$case, where the mean field potential is weaker than that for $K^{-}$, and its effects is thus comparable to that due to the Coulomb potential. We conclude that mean field effects on the $K^{-}$transverse mass spectra, especially at lower masses, are distinguishable from that due to the Coulomb potential.

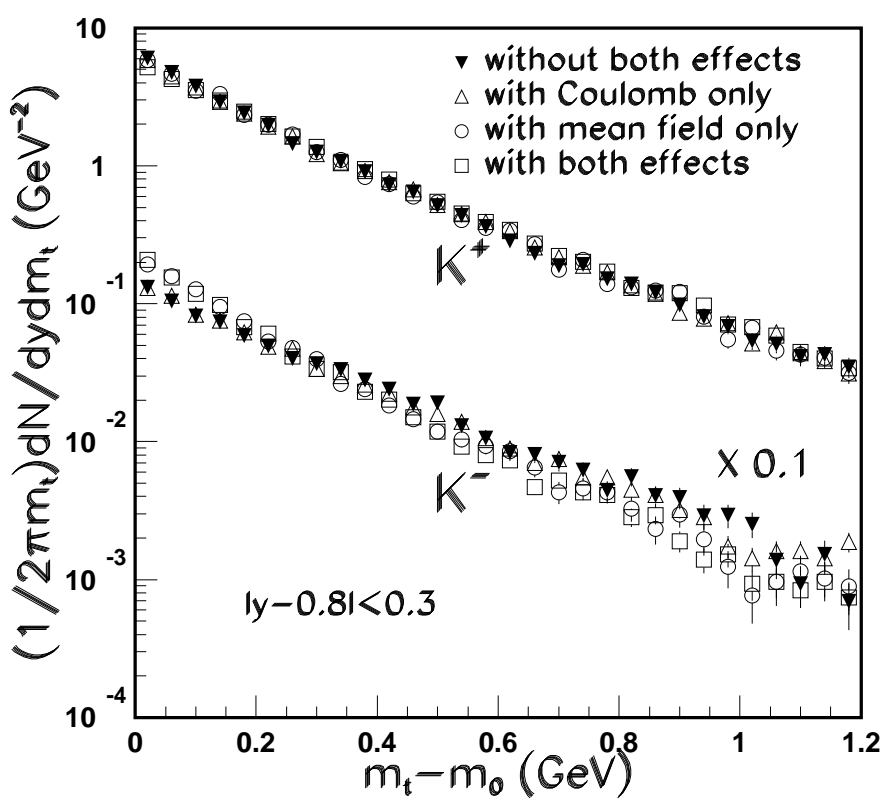

FIG. 10. Coulomb and nuclear mean field effects on transverse mass distributions of $\mathrm{K}^{+}$and $K^{-}$in the same reaction as in Fig. 4.

\section{E. Beam energy dependence of the mean field effects on antikaons}

Heavy ion collision dynamics is governed by both individual hadron-hadron collisions and mean field potentials. For baryons, mean field effects have been found to be more important in collisions at lower energies. However, it is not clear what is the beam energy dependence of the mean field effects on produced particles. To answer this question we have carried out an analysis of the rapidity distribution and transverse mass spectrum of both kaons and antikaons in the beam energy range of 2 to $16 \mathrm{GeV} /$ nucleon with and without mean field potentials. In this section, the beam energy dependence of mean field effects on antikaons is presented.

Fig. 11 shows the $K^{-}$transverse mass spectra calculated with and without the mean field potential for $\mathrm{Au}+\mathrm{Au}$ reactions at an impact parameter of $4 \mathrm{fm}$ and a beam energy of 4, 10.7 and $16 \mathrm{GeV} /$ nucleon, respectively. It is seen that the mean field effect is almost the same at all three beam energies. To be more quantitative the $K^{-}$inverse slope has been extracted by fitting the $m_{\mathrm{t}}$ spectra with exponential functions. We note that this parameter should not be identified as the temperature as effects due to collective radial flow have not been corrected. In Fig. 12, the inverse slope parameter of the $K^{-}$transverse mass spectrum 
is shown as a function of beam energy. It is seen that it increases with beam energy in both cases as one would expect. Moreover, the mean field potential reduces the $K^{-}$inverse slope parameter by about $20 \%$ in the whole beam energy range.

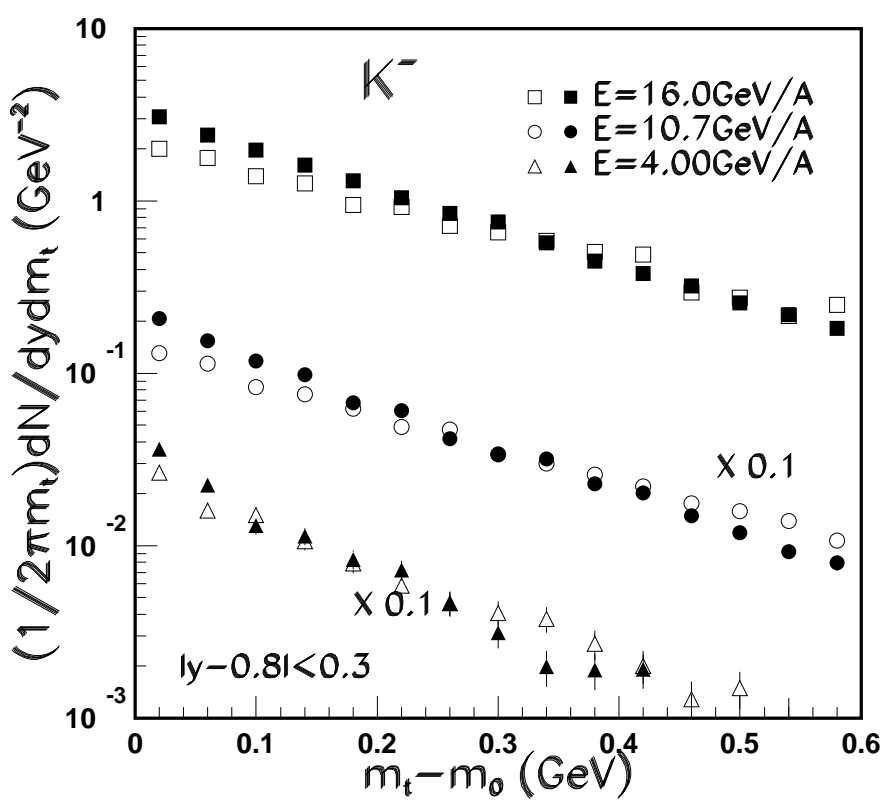

FIG. 11. Transverse mass spectra of $K^{-}$with and without the $K^{-}$mean field potential in the reaction of $\mathrm{Au}+\mathrm{Au}$ at different beam energies and an impact parameter $b=4 \mathrm{fm}$. Solid and open circles are, respectively, for the case with and without mean field potentials.

The rapidity distributions of antikaons from the same reaction as in Fig. 11 are compared in Fig. 13. It is again seen that the mean field effect on $K^{-}$rapidity distribution changes very little as the beam energy varies. We note that a similar observation has also been found for kaons [1]. We thus conclude that the mean field effects on kaons and antikaons are essentially independent of the beam energy in the energy range of 2 to $16 \mathrm{GeV} /$ nucleon. Several experimental collaborations are currently studying the beam energy dependence of particle production at the AGS, so our results can be tested in the near future.

\section{SUMMARY}

In summary, we have studied $K^{-}$production in $\mathrm{Au}+\mathrm{Au}$ collisions at different beam energies, based on an extension of the relativistic transport model ART 1.0. Since $K^{-}$has not been well treated in a previous version of this model, we have improved the model by including various reaction channels for both $K^{-}$production and scattering. Furthermore, an attractive $K^{-}$mean field potential, which is consistent with that extracted from the kaonic atom data, has been included. Our results suggest that $K^{-}$is mainly produced from mesonmeson and meson-baryon interactions. The baryon-baryon interactions are less important, contributing only one fifth of the total $K^{-}$yield. Our model is able to describe reasonably well the observed $K^{-}$rapidity and transverse momentum distributions. Furthermore, the 


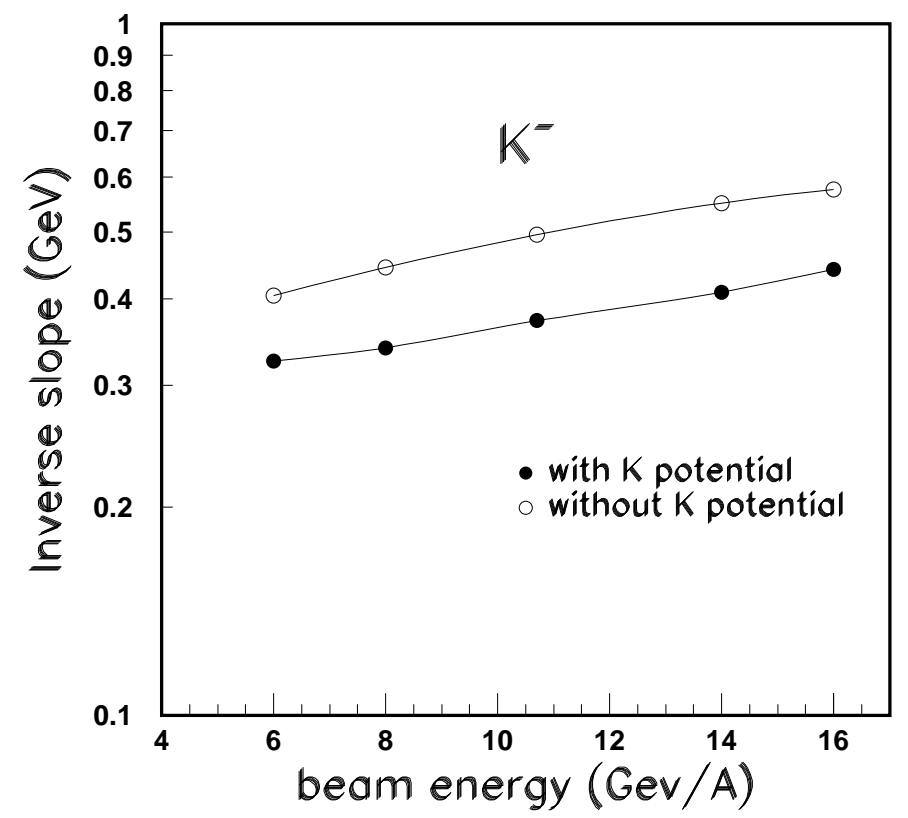

FIG. 12. Inverse slope or apparent temperature of $K^{-}$with and without $K^{-}$mean field potential in collisions of $\mathrm{Au}+\mathrm{Au}$ at different beam energies and impact parameter $b=4 \mathrm{fm}$.

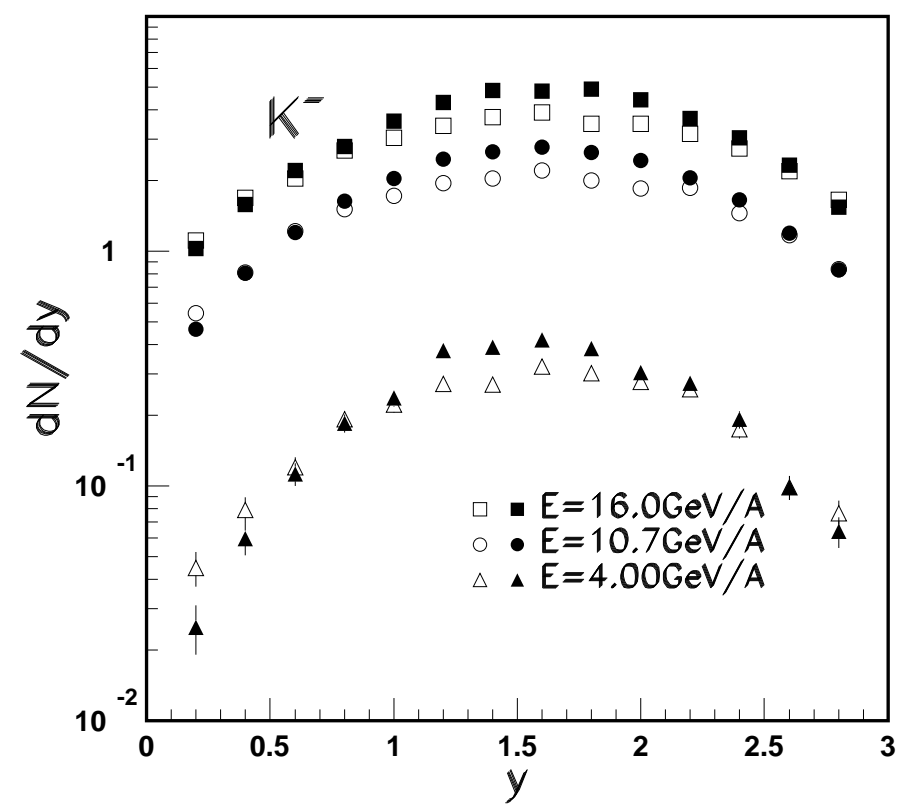

FIG. 13. Same as Fig. 11 for the rapidity distribution of $K^{-}$with and without the mean field potential. 
mean field effect is much more clearly seen on the $K^{-}$rapidity distribution and transverse mass spectrum, compared to those for $K^{+}$. Also, the $K^{+} / K^{-}$ratio as a function of transverse momentum or rapidity offers another possibility for studying the medium effects.

We have also used the model to study the $K^{-}$flow and found that due to the attractive mean field potential the $K^{-}$flow is similar to the nucleon flow. Furthermore, the medium effect on $K^{-}$flow is much stronger than that on the $K^{+}$flow, which was previously studied using the ART 1.0 model.

We have compared the effect due to the nuclear potential to that due to the Coulomb potential. Our results show that, unlike the case of $K^{+}$where the two effects are comparable, the nuclear mean field potential has a much stronger effect on $K^{-}$than the Coulomb potential. However, both nuclear and Coulomb potentials affect the $K^{-}$in a similar way, i.e., they both tend to shift the $K^{-}$from high momentum to a lower value.

We have also carried out an extensive calculation for the $K^{+}$and $K^{-}$yields, rapidity distributions and $m_{t}$ spectra at different beam energies with and without mean field potentials. We have found that for $K^{-}$medium effects are almost independent of the incident energy due to the fact that although $K^{-}$produced at lower energies are more susceptible to the influence of mean field potential, which becomes, however, weaker as the beam energy is reduced.

To conclude, it will be very useful to have more experimental data from heavy ion collisions at the AGS energies to test the predictions from our theoretical studies. Such a study will help improve our understanding of medium effects on antikaons.

\section{Acknowledgement}

We would like to thank C. Ogilvie for his interest in this study and B. Zhang for a critical reading of the manuscript. This work was supported in part by NSF Grant No. PHY-9509266 and PHY-9870038, the Robert A Welch foundation under Grant A-1358, and the Texas Advanced Research Program. 


\section{REFERENCES}

[1] B. A. Li and C. M. Ko, Phys. Rev. C 52 (1995) 2037.

[2] G. E. Brown, C. M. Ko, Z. G. Wu and L. H. Xia, Phys. Rev. Lett. 66 (1991) 2577; Phys. Rev. C 43 (1991) 1881.

[3] X. S. Fang, C. M. Ko, G. E. Brown, and V. Koch, Phys. Rev. C 47 (1993) 1678.

[4] C. M. Ko and G. Q. Li, J. Phys. G 22 (1996) 1673.

[5] G. E. Brown and M. Rho, Phys. Rev. Lett. 66 (1991) 2720.

[6] C. M. Ko, V. Koch, and C. M. Ko, Ann. Rev. Nucl. Part. Sci. 47 (1997) 505.

[7] D. B. Kaplan and A. Nelson, Phys. Lett. B 175 (1986) 57.

[8] C.-H. Lee, G. E. Brown, D.-P. Min and M. Rho, Nucl. Phys. A 585 (1995) 401.

[9] J. Schaffner, A. Gal, I. N. Mishustin, H. Stöcker and W. Greiner, Phys. Lett. B 334 (1994) 268.

[10] J. Schaffner-Bielich, I. N. Mishustin, and J. Bondorf, Nucl. Phys. A 625 (1997) 325.

[11] M. Lutz, A. Steiner and W. Weise, Phys. Lett. B 278 (1992) 29; Nucl. Phys. A 547 (1994) 755.

[12] V. Koch, Phys. Lett. B 337 (1994) 7.

[13] T. Wass, N. Kaiser, and W. Weise, Phys. Lett. B 365 (1996) 12; Phys. Lett. B 379 (1996) 34 .

[14] G. E. Brown, K. Kubodera, and M. Rho, Phys. Lett. B 192 (1987) 273.

[15] G. E. Brown and H. A. Bethe, Astrophys. J. 423 (1994) 659.

[16] G. E. Brown, C.-H. Lee, M. Rho and V. Thorsson, Nucl. Phys. A 567 (1994) 937.

[17] Proc. Work. on heavy ion physics at the AGS, Detroit, 1996, ed. C. A. Pruneau et al.

[18] C. A. Ogilvie, J. Phys. G 23 (1997) 1803; Nucl. Phys. A (1998), in press.

[19] B. A. Li and C. M. Ko, Phys. Rev. C 54 (1996) 3283.

[20] B. A. Li and C. M. Ko, Phys. Rev. C C 53 (1996) R22: C 54 (1996) 844; Nucl. Phys. A 601 (1996) 457; Phys. Rev. C 58 (1998) R1382.

[21] S. V. Efremov and E. Ya. Paryev, Z. Phys. A 348 (1994) 217.

[22] A. Sibirtsev, W. Cassing, and C. M. Ko, Z. Phys. A 358 (1997) 101.

[23] G. Q. Li, C. H. Lee, and G. E. Brown, Nucl. Phys. A 625 (1997) 372.

[24] W. Zwermann and B. Schürmann, Phys. Lett. B 145 (1984) 315.

[25] Properties and production spectra of elementary particles, ed. A. N. Diddends, H. Pilkuhn, and K. Schlüpmann (Springer-Verlag, Berlin, 1972).

[26] J. Cugnon, P. Deneye, and J. Vandermeullen, Phys. Rev. C 41 (1990) 1701.

[27] R. Mattiello, H. Sorge, H. Stöcker and W. Greiner, Phys. Rev. Lett. 63 (1989) 1459.

[28] Y. Pang et al., Phys. Rev. Lett. 68 (1992) 2743.

[29] M. Gonin, Proc. Work. on heavy ion physics at the AGS, Boston, 1993, ed. G. S. F. Stephans, S. G. Steadman, and W. L. Kehoe, p. 184.

[30] W. Cassing and E. L. Bratkovskaya, Phys. Rep. (1998), in press.

[31] G. Q. Li, C. M. Ko and B. A. Li, Phys. Rev. Lett. 74 (1995) 235; G. Q. Li and C. M. Ko, Nucl. Phys. A 594 (1995) 460.

[32] J. Gosset, et al., Phys. Rev. Lett. 62 (1989) 1251.

[33] B. A. Li, W. Bauer and G. F. Bertsch, Phys. Rev. C 44 (1991) 2095.

[34] B. A. Li, Nucl. Phys. A 570 (1994) 797.

[35] J. C. Kintner et al., Phys. Rev. Lett. 78 (1997) 4165. 
[36] C. A. Ogilvie, Advances in Nuclear Dynamics 3, ed. W. Bauer and A. Mignerey (Plenum Press,New York, 1997) p. 247. 